\title{
A Randomized Controlled Trial to Compare the Efficacy, Safety and Tolerability of Asenapine versus Olanzapine in Management of Schizophrenia
}

\author{
Arpita Maitra ${ }^{1}$, Swati Bhattacharyya ${ }^{2}$, Sabyasachi Mukhopadhyay ${ }^{3}$, Asim Kumar Mallick ${ }^{4}$, Supreeti Biswas ${ }^{1}$, \\ Om Prakash Singh ${ }^{4}$
}

${ }^{1}$ Department of Pharmacology, Burdwan Medical College, Burdwan, ${ }^{2}$ Department of Pharmacology, RG Kar Medical College, ${ }^{3}$ Department of Neonatology, AMRI Hospital, Kolkata, ${ }^{4}$ Department of Psychiatry, Burdwan Medical College, Burdwan, India

\begin{abstract}
Objective: Schizophrenia is a serious disease characterized by impairment in the perception or expression of reality, leading to occupational and social dysfunction. The use of antipsychotic medication is now universal in the first-line treatment of schizophrenia. This study was undertaken to compare the efficacy of asenapine with a standard atypical antipsychotic, olanzapine in treating this disease.

Methods: It was designed as a single blind, randomized, controlled, parallel group, single centre Phase IV trial of a newer atypical antipsychotic, asenapine versus existing standard atypical antipsychotic, olanzapine. Total 80 subjects were enrolled as per eligibility criteria.Each recruited subject received daily treatment with the trial medication (Olanzapine $10 \mathrm{mg}$ or Asenapine $10 \mathrm{mg}$ daily) for duration of 12 weeks. BPRS, CGI-S, CGI-I, Laboratory parameters and compliance was assessed and analyzed. Continuous variables were compared by $t$ test and non-parametric data was analyzed by Mann - Whitney $U$ test and Wilcoxon signed rank test. Likely categorical variables were analyzed by chi-square test or Fisher's exact test, as appropriate.

Results: The duration of schizophrenia at presentation was comparable in both the treatment groups. There was significant reduction of BPRS score between any two visits of each treatment groups. The decline in CGI-S and CGI-I scores was statistically significant $(p<0.001)$ when compared between visits of any of the both treatment arms. Adherence to treatment was excellent for all patients.

Conclusion: Newer atypical antipsychotic asenapine is more effective than standard olanzapine in reducing the symptoms of schizophrenia in this study and further larger studies are to be done.
\end{abstract}

KEY WORDS: Schizophrenia; Perception; Asenapine; Olanzapine.

\section{INTRODUCTION}

Schizophrenia is a serious disease characterized by impairment in the perception or expression of reality, leading to occupational and social dysfunction [1]. Symptoms of schizophrenia have been categorized as 'positive symptoms', which typically include delusions, hallucinations and thought disorder, and 'negative symptoms', which describe inappropriate or non-present emotion, poverty

Received: March 24, 2020 / Revised: May 14, 2020

Accepted: May 17, 2020

Address for correspondence: Swati Bhattacharyya

Department of Pharmacology, RG Kar Medical College, 1,

Khudiram Bose Sarani, Kolkata 700004, West Bengal, India

E-mail: drswatibhattacharyya@gmail.com

ORCID: https://orcid.org/0000-0002-1786-3772 of speech, and lack of motivation [2].

In India, for a population of nearly one billion people, there are an estimated four million people with schizophrenia, with different degrees of impact on some 25 million family members. Schizophrenia exposes those afflicted individuals to higher degrees of morbidity and mortality than is seen in the general population $[3,4]$.

The use of antipsychotic medication is now universal in the first-line treatment of schizophrenia. First-generation (conventional) and second-generation (atypical) antipsychotics are effective in treating the positive symptoms associated with these disorders, but efficacy for negative symptoms and cognitive dysfunction is an unmet need. Atypical ones are able to produce an antipsychotic effect

(c) This is an Open-Access article distributed under the terms of the Creative Commons Attribution Non-Commercial License (http://creativecommons.org/licenses/by-nc/4.0) which permits unrestricted non-commercial use, distribution, and reproduction in any medium, provided the original work is properly cited. 
at doses which produced little or no extra pyramidal symptoms. Olanzapine, an atypical antipsychotic introduced in 1996 [5] is now commonly used as a first-line drug in the treatment of acute symptoms of the disease, and also for long term maintenance therapy. Recent studies have shown that this drug is likely to induce weight gain and presents an increased risk for obesity-related diseases, even metabolic syndrome [6]. Indeed, in the USA, olanzapine comes with a specific warning for increased risk of diabetes mellitus [7].

Asenapine is an atypical antipsychotic approved for the treatment of schizophrenia and for the acute treatment of manic or mixed episodes of bipolar I disorder with or without psychotic features in adults. Its human receptor signature is characterized by strong affinity for serotonin receptor subtypes $\left(5-\mathrm{HT}_{1 \mathrm{~A}}, 5-\mathrm{HT}_{1 \mathrm{~B}}, 5-\mathrm{HT}_{2 \mathrm{~A}}, 5-\mathrm{HT}_{2 \mathrm{~B}}, 5-\mathrm{HT}_{2 \mathrm{C}}\right.$, $\left.5-\mathrm{HT}_{5}, 5-\mathrm{HT}_{6}, 5-\mathrm{HT}_{7}\right)$, dopamine receptor subtypes $\left(\mathrm{D}_{1}\right.$, $\left.D_{2}, D_{3}, D_{4}\right), \alpha$-adrenergic receptors, and histaminic receptors, but no appreciable affinity for muscarinic receptors. Asenapine is administered as a fast-dissolving sublingual tablet for rapid absorption through the oral mucosa $[8,9]$. In all the studies, asenapine was significantly better than placebo as measured by improvements on the positive and negative syndrome scale (PANSS) [10] total score. There is a dearth of randomized controlled trials (RCTs) comparing the efficacy and safety of asenapine with other antipsychotics in schizophrenia in Indian population. When compared to other atypical antipsychotic, there are tentative data that olanzapine may be slightly more effective. Asenapine is a novel molecule, available by sublingual route and proven for use in schizophrenia. Its efficacy has been proven over placebo and risperidone already in trials. Pubmed search showed only two head-tohead trials between asenapine vs. olanzapine till date. This paucity of evidence was the reason for conducting this trial. Thus this study was undertaken to compare the efficacy of asenapine with a standard atypical antipsychotic, olanzapine in treating this chronic disease.

\section{METHODS}

The study was designed as a single blind, randomized, controlled, parallel group, Phase IV trial of a newer atypical antipsychotic, asenapine versus existing standard atypical antipsychotic, olanzapine and was carried out at a single centre. This study was initiated after approval from the Institutional Ethics Committee and spanned over a period of 12 months since inception (BMC/PG/174/1(2)). The target sample size was 33 in each group. Considering Type I error $=0.05$, power $80 \%$, effect size $=7$ for the Brief Psychiatric Rating Scale (BPRS), standard deviation $(S D)=10$ for BPRS [11], the required sample is 33 patients in each group. These parameters were obtained from previous similar studies. Sample size was calculated with this formula, $\mathrm{n}=2\left(\mathrm{Z}_{\alpha}+\mathrm{Z}_{1-\beta}\right) 2 \sigma 2 / \Delta 2$, where $\mathrm{Z}_{\alpha}, \mathrm{Z}_{1-\beta}, \sigma, \Delta$ were respectively $1.96,0.8416,1,0.7$. After including $20 \%$ drop-out the number of patients required to be recruited in each group is 40 . All patients were scheduled for screening visit. Patients of either sex, aged between 18 to 50 years attending outpatient department (OPD) were diagnosed as schizophrenia by Psychiatrist and were selected as per eligibility criteria. Patients with severe psychosis, hepatic or renal impairment, on any other antipsychotic or antidepressant or immunosuppressant drugs, with substance abuse and pregnant females, lactating mothers were excluded. Written informed consent was taken from each recruited participant. Concomitant medication was noted and non-permitted medications were withdrawn prior to randomization of the study. Laboratory parameters including random blood sugar, lipid profile, complete hemogram, electrocardiogram (ECG) and clinical parameters like blood pressure (BP), body weight were assessed at screening visit. The baseline visit was scheduled five days after screening visit. Each recruited subject received daily treatment with the trial medication for duration of 12 weeks. Patient was advised for at least two follow ups in between the start and end of the study. Efficacy was assessed by BPRS [11], Clinical Global Impression (CGI)-S, CGI-I [12-14], where safety was assessed by evaluating clinical and laboratory parameters (complete hemogram, Random blood sugar, lipid profile) at screening and at end of study visit. The BPRS is a rating scale which a clinician or researcher may use to measure psychiatric symptoms such as depression, anxiety, hallucinations and unusual behavior. Each symptom is rated $1-$ 7 and a total of 24 symptoms are scored. 1-absent, 2-very mild, 3-mild, 4-moderate, 5-moderately severe, 6 -severe, 7-extremely severe. A total 18 symptoms are assessed in this method. CGI scale is treatment response ratings which should take account of both therapeutic efficacy and treatment-related adverse events. Each component of the CGI is rated separately; the instrument does not yield a 
global score.

\section{Severity of IIIness (CGI-S)}

Considering your total clinical experience with this particular population, how mentally ill is the patient at this time?

$0=$ Not assessed

$1=$ Normal, not at all ill

2 = Borderline mentally ill

$3=$ Mildly ill

$4=$ Moderately ill

5 = Markedly ill

$6=$ Severely ill

$7=$ Among the most extremely ill patients

\section{Global Improvement (CGI-I)}

Rates total improvement whether or not; it is due entirely to drug treatment. Compared to his condition at admission to the project, how much has he changed?

$0=$ Not assessed

$1=$ Very much improved

2 = Much improved

3 = Minimally improved

$4=$ No change

5 = Minimally worse

$6=$ Much worse

7 = Very much worse

All these three scales are globally accepted and used widely for evaluating patients with Schizophrenia. Many randomized controlled trials dealing with different antipsychotic drugs have demonstrated usage of these scales for efficacy analysis.

After completing baseline assessment, subjects were randomized to one of the two study group medications in blocks of 10 using a computer generated random number list.

Patients were divided in two groups-Group A (Asenapine $10 \mathrm{mg}$ total daily dose) and Group B (Olanzapine $10 \mathrm{mg}$ total daily dose) and both the groups received either one of the following medications. The starting dose was decided by the concerned psychiatrist, depending on the severity of illness. Up titration, if needed, was permitted only once during the course of the study, within the maximum limits stated here. Olanzapine was supplied from hospital. Asenapine was bought by the investigator herself and supplied free of cost to patients. There was no conflict of interest. The medications were kept in boxes coded as
'Drug A' (Asenapine) or 'Drug B' (Olanzapine) with respect to the identity of the medication. The blinding code was kept in the knowledge of the Research Supervisor. The single blinding (investigator blinding) was done by ensuring the trial medications were dispensed by OPD nurse. During follow up the patient was asked to report first to the OPD nurse for pill count. Follow up visits were scheduled at 4 and 8 weeks, with provision for additional interim follow ups, if needed, for assessing adverse events. The final end-of-treatment visit was scheduled at 12 weeks. Efficacy and laboratory parameters were further assessed in each follow up and end of study visit. Compliance was assessed by the traditional pill count method. It was deemed to be excellent if not more than $10 \%$ of schedule doses were missed, good if not more than $20 \%$ were missed, fair if not more than $30 \%$ were missed, and poor for any situation worse than fair. All data were recorded in case record form for each case.

Data were analyzed with the help of SPSS version 21 (IBM Co., Armonk, NY, USA) [15]. Continuous variables were compared between groups by independent samples $t$ test and within group by paired $t$ test. Mann - Whitney $U$ test and Wilcoxon signed rank test was carried out for unpaired and paired non-parametric data. Categorical data were compared between groups by chi-square test or Fisher's exact test, as appropriate. Friedman's ANOVA (by post hoc analysis with Multiple Wilcoxon Signed Ranks test with a Bonferroni correction) or repeated measures ANOVA (followed by post hoc Bonferroni correction) were done in case of within group comparison in case of non-parametric data or normally distributed data respectively. Efficacy analysis was done on modified intentionto-treat basis for the patients reporting for at least two post-baseline follow-up visits. This strategy was followed based on some similar antipsychotic drug trials. Missing values were dealt with using the last observation carried forward strategy. Among the 40 randomized patients of Olanzapine group, 2 patients were excluded from analysis due lost to follow up and none fulfilled modified intention-to-treat (mITT) criteria. Similarly 1 patient from Asenapine group was excluded from analysis.

Per protocol analysis strategy was not taken to minimize the chance of attrition and protocol deviation. Results are based on mITT analysis, where 3 patients were excluded due to not attending OPD after receiving baseline treatment. 


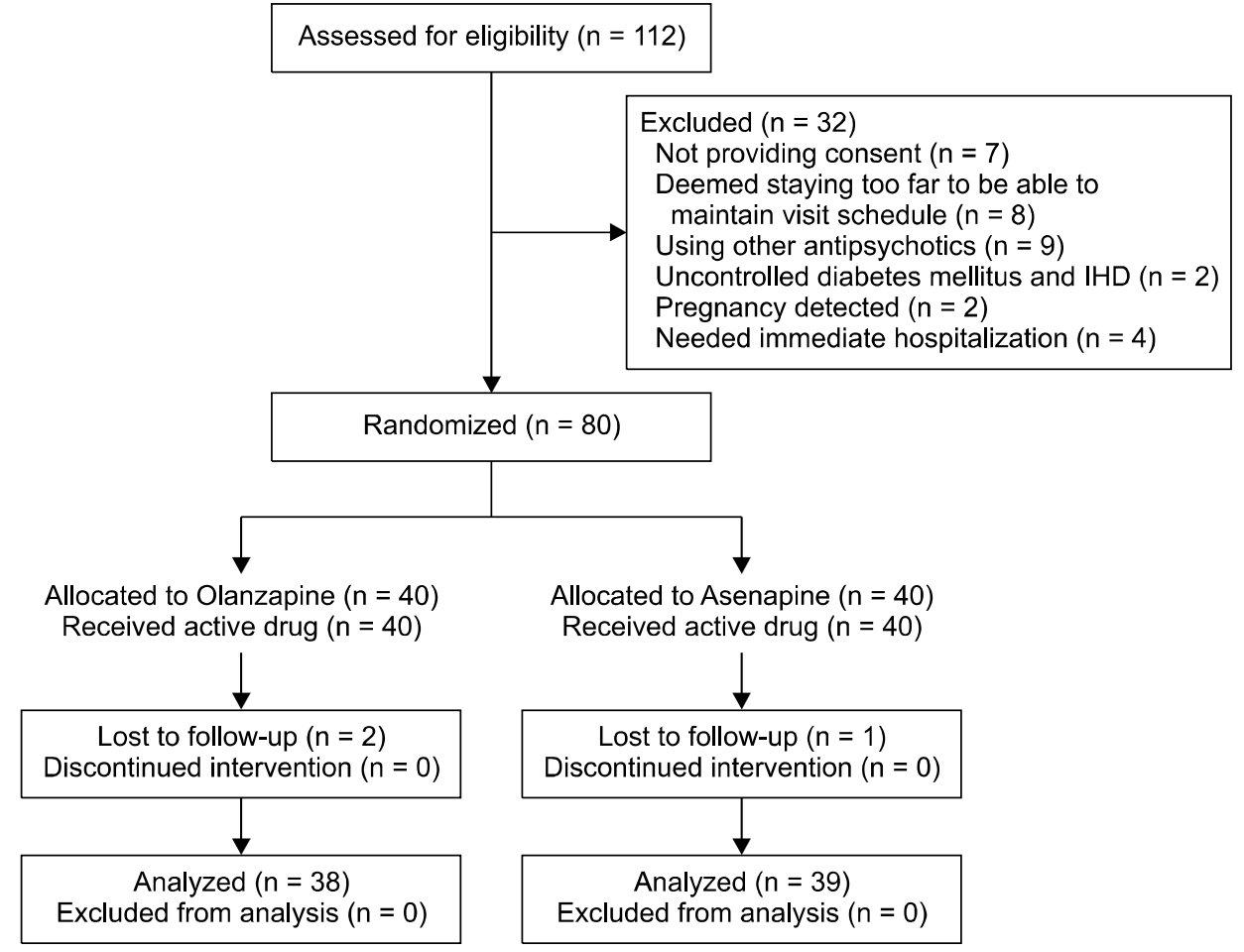

Fig. 1. CONSORT diagram of subjects. $\mathrm{IHD}$, ischemic heart disease.

\section{RESULTS}

Out of 112 screened patients them 80 (71.4\%) were recruited- 40 in each group. Of these, 77 patients fulfilled modified-intention-to-treat criteria [16], 39 in asenapine group and 38 in olanzapine group. 3 patients were lost to follow-up, of which 1 from asenapine group and 2 from olanzapine group. Reason of lost to follow up was common in all 3 patients. They could not attend the OPD further after receiving baseline treatment due to lack of social support. Family members informed about their follow up treatment in nearby hospitals on as and when required basis (Fig. 1)

Baseline characteristics of all study subjects are depicted in Table 1. Gender distribution was nearly equal and the patients were in their thirties, indicating preponderance to younger age groups.

The duration of schizophrenia at presentation was comparable in both the treatment groups. In Asenapine group the mean duration was $21.15 \pm 5.48$ months where in Olanzapine group it was $22.37 \pm 5.29$ months.

\section{Efficacy Parameters}

\section{Changes in Brief Psychiatric Rating Scale score}

The changes in BPRS score are depicted in Table 2 and Figure 2. The BPRS scores were declining over the treatment period for both the groups. This decline was statistically significant $(p<0.001)$ when compared between visits of any of the both treatment arms. In case of between group comparison, it is evident that there was no statistically significant difference in BPRS score in baseline visit (t [75] $=1.080, p=0.284)$ and 1 st follow-up visit $(\mathrm{t}[75]=$ $-0.661, p=0.511)$; but there was statistically significant difference in BPRS score in 2nd follow-up ( $\mathrm{t}$ [75] $=-7.281$, $p<0.001$ ) and end follow-up visit (t [75] $=-5.185, p<$ 0.001).

A repeated measures ANOVA with a GreenhouseGeisser correction determined that mean BPRS score differed statistically significant in between visits ( $F$ [1.691, $126.842]=29.232, p<0.001)$. Post hoc tests using the Bonferroni correction revealed that there was significant reduction of BPRS score between any two visits of each treatment groups. 
Table 1. Demographic profile of study population

\begin{tabular}{|c|c|c|c|}
\hline Category & $\begin{array}{c}\text { Asenapine } \\
\text { group }(n=39)\end{array}$ & $\begin{array}{c}\text { Olanzapine } \\
\text { group }(n=38)\end{array}$ & $\begin{array}{c}p \text { value } \\
\text { (between } \\
\text { groups) }\end{array}$ \\
\hline \multicolumn{4}{|l|}{ Age (yr) } \\
\hline Range & $18-50$ & $18-50$ & 0.444 \\
\hline $\begin{array}{l}\text { Mean } \pm \text { standard } \\
\text { deviation }\end{array}$ & $31.51 \pm 5.86$ & $32.66 \pm 7.14$ & \\
\hline \multicolumn{4}{|l|}{ Sex } \\
\hline Male & $22(56.4)$ & $18(47.4)$ & 0.497 \\
\hline Female & $17(43.6)$ & $20(52.6)$ & \\
\hline \multicolumn{4}{|l|}{ Residence } \\
\hline Urban & $20(51.3)$ & $13(34.2)$ & 0.169 \\
\hline Rural & 19 (48.7) & 25 (65.8) & \\
\hline \multicolumn{4}{|l|}{ Literacy } \\
\hline Illiterate & $3(7.7)$ & $3(7.9)$ & 0.171 \\
\hline Primary & $6(15.4)$ & $11(28.9)$ & \\
\hline Secondary & $17(43.6)$ & $8(21.1)$ & \\
\hline $\begin{array}{l}\text { Higher secondary } \\
\text { and above }\end{array}$ & $13(33.3)$ & $16(42.1)$ & \\
\hline \multicolumn{4}{|l|}{ Occupation } \\
\hline Student & $6(15.4)$ & $2(5.3)$ & 0.307 \\
\hline $\begin{array}{l}\text { Home worker/ } \\
\text { housewife }\end{array}$ & $9(23.1)$ & $12(31.6)$ & \\
\hline Agricultural worker & $12(30.8)$ & $7(18.4)$ & \\
\hline $\begin{array}{l}\text { Non-agricultural } \\
\text { outdoor worker }\end{array}$ & $6(15.4)$ & $10(26.3)$ & \\
\hline $\begin{array}{l}\text { Non-agricultural } \\
\text { indoor worker }\end{array}$ & $6(15.4)$ & $7(18.4)$ & \\
\hline
\end{tabular}

Values are presented as number (\%).

$p$ value is from Student's independent $t$ test for age, and from chi-square test for sex distribution, residence, literacy, occupation.

Table 2. Changes in BPRS score over 12 weeks

\begin{tabular}{lccc}
\hline \multicolumn{1}{c}{ BPRS } & $\begin{array}{c}\text { Asenapine } \\
\text { group }(\mathrm{n}=39)\end{array}$ & $\begin{array}{c}\text { Olanzapine } \\
\text { group }(\mathrm{n}=38)\end{array}$ & $\begin{array}{c}p \text { value } \\
\text { (between } \\
\text { groups) }\end{array}$ \\
\hline Baseline & $61.18 \pm 4.29$ & $60.13 \pm 4.23$ & 0.284 \\
1st follow-up & $54.00 \pm 3.33$ & $54.53 \pm 3.65$ & 0.511 \\
2nd follow-up & $38.10 \pm 3.06$ & $44.11 \pm 4.11$ & $<0.001$ \\
End follow-up & $27.10 \pm 3.57$ & $31.61 \pm 4.04$ & $<0.001$ \\
\hline
\end{tabular}

Values are presented as mean \pm standard deviation. BPRS, Brief Psychiatric Rating Scale.

$p$ value for between group comparisons is from Independent-Samples $t$ test. $p$ value for within group comparison between the four visits done by Repeated Measures ANOVA followed by post hoc Bonferroni correction.

\section{Changes in the Clinical Global Impression score for severity of illness}

It is evident that the CGI-S scores (shown in Table 3 and Fig. 3) were declining over the treatment period for both the groups. This decline was statistically significant $(p<$ 0.001 ) when compared between visits of any of the both
Estimated marginal means

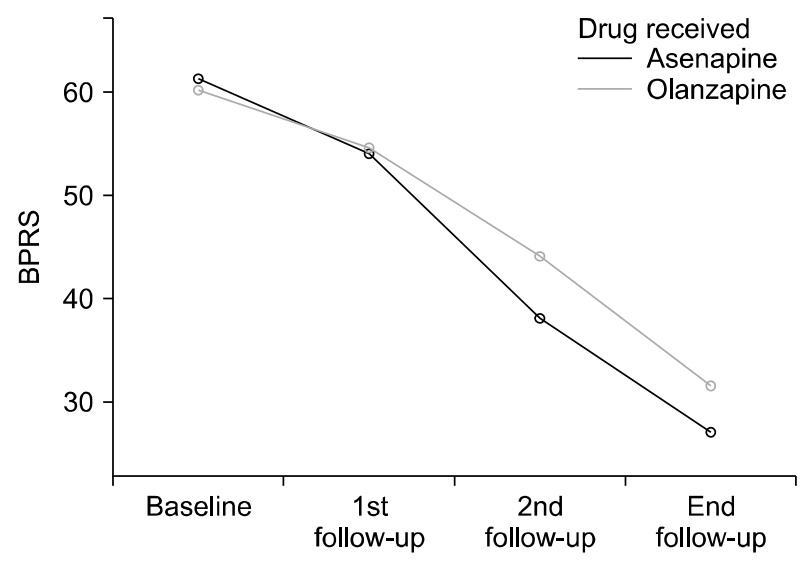

Fig. 2. Line diagram of Brief Psychiatric Rating Scale (BPRS) score in different visits in two treatment arms over 12 weeks.

Table 3. Changes in CGI-S score over 12 weeks

\begin{tabular}{|c|c|c|c|}
\hline CGI-S & $\begin{array}{c}\text { Asenapine } \\
\text { group }(n=39)\end{array}$ & $\begin{array}{c}\text { Olanzapine } \\
\text { group }(\mathrm{n}=38)\end{array}$ & $\begin{array}{c}p \text { value } \\
\text { (between } \\
\text { groups) }\end{array}$ \\
\hline Baseline & $\begin{array}{c}5.13 \pm 0.61 \\
5(5-6)\end{array}$ & $\begin{array}{c}5.03 \pm 0.59 \\
5(5-5)\end{array}$ & 0.452 \\
\hline 1st follow-up & $\begin{array}{c}4.41 \pm 0.50 \\
4(4-5)\end{array}$ & $\begin{array}{c}4.42 \pm 0.50 \\
4(4-5)\end{array}$ & 0.924 \\
\hline 2nd follow-up & $\begin{array}{c}2.87 \pm 0.41 \\
3(3-3)\end{array}$ & $\begin{array}{c}3.45 \pm 0.50 \\
3(3-4)\end{array}$ & $<0.001$ \\
\hline End follow-up & $\begin{array}{c}1.72 \pm 0.51 \\
2(1-2)\end{array}$ & $\begin{array}{c}2.16 \pm 0.49 \\
2(2-2)\end{array}$ & $<0.001$ \\
\hline
\end{tabular}

Values are presented as mean \pm standard deviation or median (interquartile range).

CGI-S, Clinical Global Impressions-Severity of Illness scale.

$p$ value for between group comparisons is from Mann-Whitney $U$ test. $p$ value for within group comparison between the four visits done by Friedman's ANOVA followed by post hoc analysis with Multiple Wilcoxon Signed Ranks test with a Bonferroni correction.

treatment arms. In case of between group comparisons, there was no statistically significant difference in CGI-S score in baseline visit and 1st follow-up visit; but there was statistically significant difference in CGI-S score in 2nd follow-up and end follow-up visit.

After doing Friedman's ANOVA, it was seen that there were significant differences in CGI-S scores depending on different visits in each treatment groups (In case of Asenapine group; $\chi^{2}[4]=151.793, p<0.001$; and in case of Olanzapine group; $\chi^{2}[4]=145.867, p<0.001$ ). Post hoc analysis with Multiple Wilcoxon Signed Ranks test with a Bonferroni correction applied, resulting in a significance level set at $p$ (0.001). There were statistical 


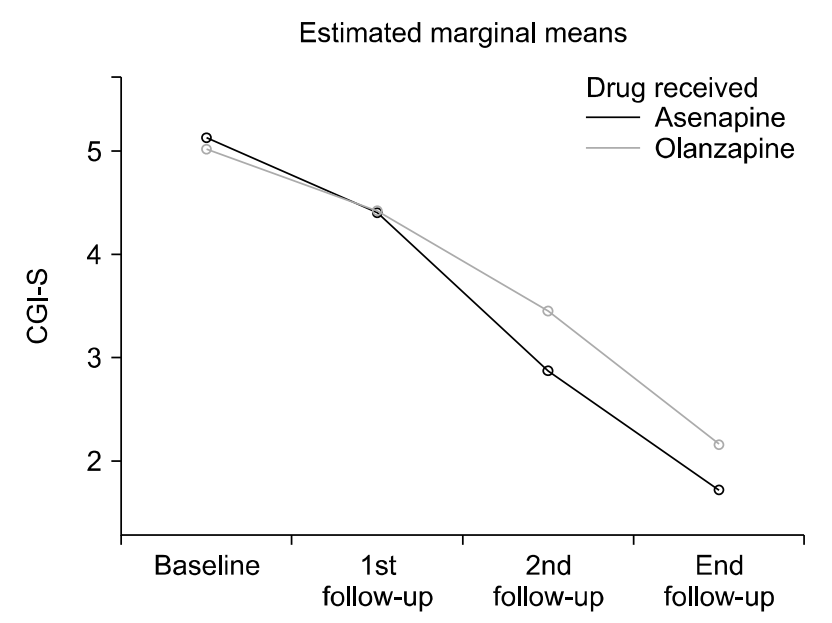

Fig. 3. Line diagram of Clinical Global Impressions-Severity of Illness scale (CGI-S) score in different visits in two treatment arms over 12 weeks.

significant differences in CGI-S scores in all possible pairing of visits.

From Mann - Whitney $U$ test it can be concluded that differences in CGI-S scores in the both treatment groups were not statistically significant at baseline $(U=678, p=$ $0.452) \& 1$ st follow-up $(U=733, p=0.924)$ visits. But the same were statistically significant at 2 nd follow-up $(\mathrm{U}=$ $365, p<0.001) \&$ end follow-up $(U=462, p<0.001)$ visits.

\section{Changes in the Clinical Global Impression score for global improvement}

CGI-I score was not assessed at baseline visit. The changes in CGI-I score are depicted in Table 4 and Figure 4. The CGI-I scores were declining over the treatment period for both the groups. This decline was statistically significant $(p<0.001)$ when compared between visits of any of the both treatment arms. In case of between group comparisons, there was no statistically significant difference in CGI-I score in 1st follow-up visit; but there was statistically significant difference in CGI-I score in 2nd follow-up and end follow-up visits.

After Friedman's ANOVA, there were significant differences in CGI-I scores depending on different visits in each treatment groups (In case of Asenapine group; $\chi^{2}[2]=$ 76.588, $p<0.001$; and in case of Olanzapine group; $\left.\chi^{2}[4]=72.375, p<0.001\right)$. Post hoc analysis with Multiple Wilcoxon Signed Ranks test with a Bonferroni correction applied, resulting in a significance level set at $p$
Table 4. Changes in CGI-I score over 12 weeks

\begin{tabular}{lccc}
\hline \multicolumn{1}{c}{ CGI-I } & $\begin{array}{c}\text { Asenapine } \\
\text { group }(\mathrm{n}=39)\end{array}$ & $\begin{array}{c}\text { Olanzapine } \\
\text { group }(\mathrm{n}=38)\end{array}$ & $\begin{array}{c}p \text { value } \\
\text { (between } \\
\text { groups) }\end{array}$ \\
\hline Baseline & $0 \pm 0$ & $0 \pm 0$ & - \\
1st follow-up & $0(0-0)$ & $0(0-0)$ & 0.920 \\
2nd follow-up & $2.87 \pm 0.50$ & $4.45 \pm 0.50$ & \\
& $3(4-5)$ & $4(4-5)$ & $<0.001$ \\
End follow-up & $1.72 \pm 0.51$ & $3.45 \pm 0.50$ & $3(3-4)$ \\
& $2(1-2)$ & $2.16 \pm 0.49$ & $<0.001$ \\
\hline
\end{tabular}

Values are presented as mean \pm standard deviation or median (interquartile range).

CGI-I, Clinical Global Impressions-Improvement scale.

$p$ value for between group comparisons is from Mann-Whitney $U$ test. $p$ value for within group comparison between the four visits done by Friedman's ANOVA followed by post hoc analysis with Multiple Wilcoxon Signed Ranks test with a Bonferroni correction.

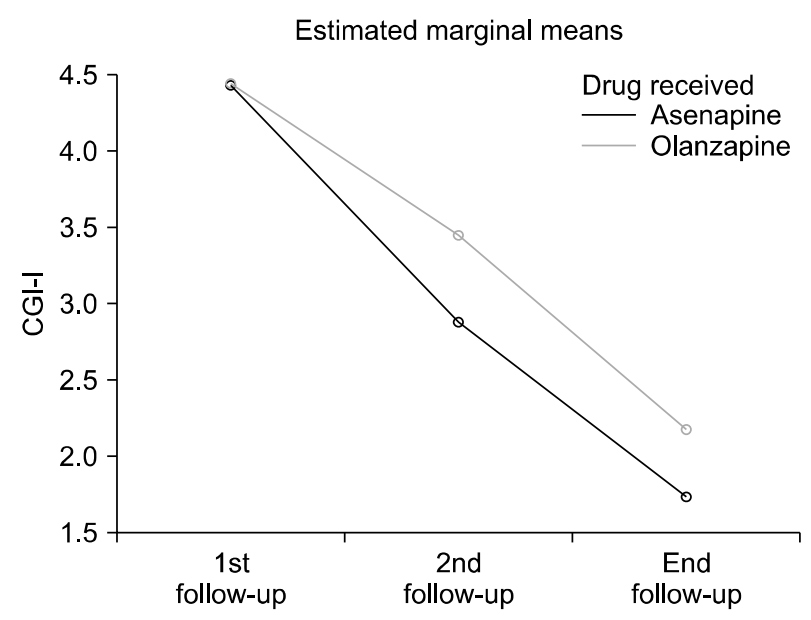

Fig. 4. Line diagram of Clinical Global Impressions-Improvement scale (CGI-I) score in different visits in two treatment arms over 12 weeks.

(0.001). There were statistical significant differences in CGI-I scores in all possible pairing of visits.

From Mann - Whitney $U$ test it can be concluded that difference in CGI-I scores in the both treatment groups was not statistically significant at 1 st follow-up $(\mathrm{U}=$ 732.5, $p=0.920$ ) visit. But the same were statistically significant at 2nd follow-up $(U=365.5, p<0.001)$ \& end follow-up $(U=462, p<0.001)$ visits.

Compliance assessment covered only those subjects who completed the study as per protocol. The end-of-study compliance assessment indicates adherence to treatment was excellent for all patients in both treatment groups. Changes in laboratory parameters were comparable in 
Table 5. Changes in laboratory parameters in the two treatment groups

\begin{tabular}{|c|c|c|c|}
\hline Category & Asenapine group $(n=39)$ & Olanzapine group $(\mathrm{n}=38)$ & $p$ value (between groups) \\
\hline \multicolumn{4}{|l|}{ Haemoglobin (g/dl) } \\
\hline Baseline & $11.68 \pm 1.17$ & $11.32 \pm 1.16$ & 0.173 \\
\hline End follow-up & $11.99 \pm 0.99$ & $11.67 \pm 1.04$ & 0.166 \\
\hline Before-after $p$ value & 0.006 & $<0.001$ & \\
\hline \multicolumn{4}{|l|}{ TLC (cells/ $\mu \mathrm{l})$} \\
\hline Baseline & $7,710.26 \pm 1,382.37$ & $7,150.00 \pm 1,409.95$ & 0.082 \\
\hline End follow-up & $7,635.89 \pm 1,335.05$ & $7,386.84 \pm 1,488.55$ & 0.442 \\
\hline Before-after $p$ value & 0.809 & 0.481 & \\
\hline \multicolumn{4}{|l|}{ Neutrophils (\%) } \\
\hline Baseline & $63.41 \pm 9.16$ & $62.74 \pm 7.99$ & 0.732 \\
\hline End follow-up & $65.36 \pm 7.21$ & $62.97 \pm 8.57$ & 0.190 \\
\hline Before-after $p$ value & 0.232 & 0.889 & \\
\hline \multicolumn{4}{|l|}{ Eosinophils (\%) } \\
\hline Baseline & $5.56 \pm 2.29$ & $4.58 \pm 2.04$ & 0.06 \\
\hline End follow-up & $4.08 \pm 1.68$ & $4.21 \pm 1.54$ & 0.539 \\
\hline Before-after $p$ value & 0.002 & 0.630 & \\
\hline \multicolumn{4}{|l|}{ Lymphocytes (\%) } \\
\hline Baseline & $28.67 \pm 8.21$ & $27.92 \pm 7.59$ & 0.680 \\
\hline End follow-up & $30.23 \pm 7.41$ & $30.74 \pm 8.43$ & 0.780 \\
\hline Before-after $p$ value & 0.381 & 0.078 & \\
\hline \multicolumn{4}{|l|}{ Basophils (\%) } \\
\hline Baseline & 0 & $0.026 \pm 0.162$ & 0.311 \\
\hline End follow-up & 0 & $0.026 \pm 0.162$ & 0.311 \\
\hline Before-after $p$ value & 1.00 & 1.00 & \\
\hline \multicolumn{4}{|l|}{ Monocytes (\%) } \\
\hline Baseline & $2.23 \pm 1.16$ & $2.03 \pm 1.44$ & 0.439 \\
\hline End follow-up & $2.00 \pm 1.10$ & $1.89 \pm 1.06$ & 0.693 \\
\hline Before-after $p$ value & 0.279 & 0.728 & \\
\hline \multicolumn{4}{|l|}{ ESR (mm in $1 \mathrm{st} \mathrm{hr)}$} \\
\hline Baseline & $15.64 \pm 11.88$ & $20.29 \pm 15.17$ & 0.238 \\
\hline End follow-up & $16.49 \pm 12.18$ & $15.03 \pm 11.97$ & 0.443 \\
\hline Before-after $p$ value & 0.856 & 0.118 & \\
\hline \multicolumn{4}{|l|}{ Cholesterol (mg \%) } \\
\hline Baseline & $146.72 \pm 13.51$ & $142.97 \pm 13.28$ & 0.397 \\
\hline End follow-up & $146.67 \pm 11.25$ & $156.11 \pm 13.43$ & 0.001 \\
\hline Before-after $p$ value & 0.872 & $<0.001$ & \\
\hline \multicolumn{4}{|l|}{ Triglyceride (mg \%) } \\
\hline Baseline & $110.33 \pm 23.06$ & $107.66 \pm 22.04$ & 0.610 \\
\hline End follow-up & $137.03 \pm 22.42$ & $136.92 \pm 21.09$ & 0.687 \\
\hline Before-after $p$ value & $<0.001$ & $<0.001$ & \\
\hline \multicolumn{4}{|l|}{ HDL cholesterol (mg \%) } \\
\hline Baseline & $54.44 \pm 6.35$ & $54.53 \pm 7.24$ & 0.927 \\
\hline End follow-up & $51.82 \pm 4.03$ & $52.29 \pm 4.39$ & 0.452 \\
\hline Before-after $p$ value & 0.019 & 0.049 & \\
\hline \multicolumn{4}{|l|}{ LDL cholesterol (mg \%) } \\
\hline Baseline & $80.05 \pm 6.19$ & $83.89 \pm 7.89$ & 0.034 \\
\hline End follow-up & $94.05 \pm 10.62$ & $96.60 \pm 10.06$ & 0.237 \\
\hline Before-after $p$ value & $<0.001$ & $<0.001$ & \\
\hline \multicolumn{4}{|l|}{ VLDL cholesterol (mg \%) } \\
\hline Baseline & $19.33 \pm 4.19$ & $19.42 \pm 4.36$ & 0.988 \\
\hline End follow-up & $29.59 \pm 4.68$ & $31.16 \pm 4.45$ & 0.169 \\
\hline Before-after $p$ value & $<0.001$ & $<0.001$ & \\
\hline \multicolumn{4}{|c|}{ Random blood glucose $(\mathrm{mg} / \mathrm{dl})$} \\
\hline Baseline & $89.44 \pm 15.51$ & $94.34 \pm 15.01$ & 0.067 \\
\hline End follow-up & $95.00 \pm 19.89$ & $92.79 \pm 17.08$ & 0.698 \\
\hline Before-after $p$ value & 0.132 & 0.0576 & \\
\hline
\end{tabular}

Values are presented as mean \pm standard deviation.

TLC, total leucocyte count; ESR, erythrocyte sedimentationrate; HDL, high density lipoprotein; LDL, low density lipoprotein; VLDL, very low density lipoprotein.

$p$ value for between group comparison is from Mann - Whitney $U$ test, whereas for within group before-after comparison is from Wilcoxon Signed Rank test in case of Eosinophil, Basophil, Monocyte and ESR; and in case of others, between group comparison is from Student's unpaired $t$ test, whereas within group before-after comparison is from Student's paired $t$ test. 
both the groups.

\section{Safety Assessment}

Adverse events recorded included treatment-emergent events reported spontaneously by subjects at any time during the 12 week treatment, serious events reported for up to 4 weeks after completing the scheduled course of study medication, those elicited as clinical signs by the investigators during the scheduled visits and adverse laboratory test results.

\section{Laboratory Parameters for Safety Determination}

In case of hematological safety parameter, there was statistically significant increase in hemoglobin level in both treatment arms but not statistically significant in case of between group studies. There was also statistically significant decrease was eosinophil count at end follow up visit in asenapine group but no such thing observed in olanzapine group. Other hematological parameters remained statistically not significant in comparison of within (before-after) or between groups. In case of biochemical parameters there was statistically significant increase of serum cholesterol in olanzapine group which was also significant in between group comparison. There were also statistically significant increase in serum triglyceride, serum low density lipoprotein (LDL) cholesterol, and serum very low density lipoprotein (VLDL) cholesterol; and statistically significant decrease in serum high density lipoprotein (HDL) cholesterol (before-after) in both treatment groups. But in case of between group comparisons the parameters showed no statistical significant differences. However the mean values of lipid profile were within normal range. No such relation was seen in case of random blood glucose. These changes are depicted in Table 5.

ECG was done to each patient during baseline and end of the treatment. No abnormalities detected to any patients before or after treatment in both the treatment groups.

An adverse event was to be deemed serious if it was fatal, life-threatening, causing significant disability, impairment or causing prolonged hospitalization, or required intervention to prevent any of these outcomes.

During the 12 week study period a total of 68 subjects were suspected of having at least one adverse drug reaction. On causality assessment, 12 of these 68 cases
Table 6. Individual suspected adverse drug reactions

\begin{tabular}{lccc}
\hline \multicolumn{1}{c}{ Adverse event } & $\begin{array}{c}\text { Asenapine } \\
\text { group } \\
(\mathrm{n}=39)\end{array}$ & $\begin{array}{c}\text { Olanzapine } \\
\text { group } \\
(\mathrm{n}=38)\end{array}$ & $\begin{array}{c}p \text { value } \\
\text { (between } \\
\text { groups })\end{array}$ \\
\hline Insomnia & 8 & 4 & 0.227 \\
Somnolence & 7 & 4 & 0.352 \\
Nausea & 2 & 7 & 0.087 \\
Tremor & 2 & 4 & 0.431 \\
Anxiety & 5 & 5 & 1.000 \\
Headache & 4 & 0 & 0.115 \\
Vomiting & 1 & 6 & 0.056 \\
Constipation & 0 & 2 & 0.240 \\
Weight gain & 3 & 10 & 0.675 \\
Increased salivation & 0 & 1 & 0.494 \\
Akathisia & 0 & 2 & 0.240 \\
\hline
\end{tabular}

$p$ value is from chi-sqare test in case of insomnia and somnolence and from Fisher's exact test in case of others.

(17.65\%) were considered to have insufficient evidence. Overall, out of 77 treatment-emergent adverse events from 56 subjects: 23 subjects $(57.5 \%$ of the 40 randomized) in the asenapine arm and 33 subjects $(82.5 \%$ of the randomized) in the olanzapine arm reported at least one event. This difference was statistically significant ( $p=$ 0.006 , by chi-square test) (Table 6)

\section{DISCUSSION}

Schizophrenia is a functionally and socially debilitating chronic psychiatric illness [17]. Beginning in early adulthood, schizophrenia typically causes a dramatic, lifelong impairment in social and occupational functioning. From a public health standpoint, the costs of treatment and lost productivity make this illness one of the most expensive disorders in medicine. This highlights the fact that the therapy of this disease should be long term and the cost of treatment should be bearable in general population.

Second generation antipsychotics are the best options when efficacy, safety and tolerability profiles are concerned $[18,19]$ and this study is a humble effort to find a new option.

The study subjects were assessed in four visits which helped thorough assessment of the patients regarding efficacy.

The study showed that most of the patients were in their thirties and nearly equally distributed in case of gender distribution. Previous studies in other parts of the globe also found a greater incidence of schizophrenia in their thir- 
ties, though males were shown to be suffering more from the disease $[20,21]$. The result of the above study was in contrast to our study. This difference can be due to a small sample size in our study, which did not reflect actual disease burden in our population. Other demographic profiles were generally comparable in both treatment arms.

The mean duration of schizophrenia symptoms were for about 21 months in both the treatment arms. The vital signs recorded at baseline were analogous in both the treatment groups. The laboratory parameters at baseline were within normal limits in both the groups.

Primary effectiveness variable for this study was BPRS score [11]. This scale describes the patient's condition by evaluating different symptoms (including positive and negative symptoms). The results of the present study showed that there was significant improvement in BPRS score in the asenapine group than the olanzapine treatment group over 12 weeks period. Also, the scores decreased from baseline significantly during all the follow-ups in both the treatment arms showing both the antipsychotics were quite effective in curing symptoms of schizophrenia. However, asenapine seemed to be more effective than olanzapine in our study. It was also found that significant reduction in BPRS score was evident after 1 st follow-up (4 weeks onwards) indicating rapid reduction of symptoms which goes in favor of use of atypical antipsychotics. Asenapine caused significant reduction in BPRS score more from 2nd follow-up (8 weeks) onwards than olanzapine.

Considering the secondary effectiveness variables, there were significant decrease $(p<0.001)$ in the CGI-S scores and CGI-I scores [12-14] from baseline in both the treatment groups. When the groups were compared to each other, the asenapine arm showed significantly better improvement of both the scales $(p<0.001)$ from 2 nd follow-up visits to end follow-up visit. Those observations reflected that both the drugs are worth prescribing in schizophrenia.

Efficacy of asenapine has been evaluated in multiple clinical trials. It was observed in the studies that, asenapine significantly improved symptoms in adult patients requiring treatment for acute schizophrenia. It was observed that, asenapine demonstrated significantly better efficacy than placebo and was as efficacious as the active comparators (haloperidol, risperidone, olanzapine) [21,22]. Of the 6 short-term studies, four studies tested the efficacy of asenapine within the recommended dose range of $5-$ $10 \mathrm{mg}$ twice daily. The efficacy of asenapine has been well established in 2 of these 4 trials [20,22]. The primary endpoint in the trials was the least squares mean change from baseline in PANSS total score. Secondary outcome measures included the weekly change from baseline in CGI-S score and weekly changes in positive, negative and general psychopathology subscale scores.

Potkin et al. [22] evaluated the efficacy of asenapine against risperidone in a RCT assessing the change in PANSS total score. Both asenapine and risperidone significantly reduced all the scores. Improvement in least squares mean PANSS total score in asenapine recipients was evident after 2 weeks of treatment ( $p<0.05$ vs. placebo) [22]. Both asenapine and risperidone treatments was associated with significantly greater decrease in CGI-S sub score which was evident from week 4 onwards [22]. This observation was also seen in the present study where primary efficacy variable was BPRS score and secondary efficacy variables were CGI-S and CGI-I score. Similar observations were found by Kane et al. [20] where the efficacy of asenapine $5 \mathrm{mg}$, and $10 \mathrm{mg}$ twice daily were evaluated with an acute exacerbation of schizophrenia in RCT compared with placebo. In study of Kane et al. [20] the results were similar when compared with placebo. In case of CGI-I score, the proportion of CGI-I responders were statistically significant in the asenapine group [20].

Schoemaker et al. [21] compared the efficacy of long term asenapine versus olanzapine in patients with schizophrenia or schizoaffective disorder. Here it was observed that changes (mean \pm SD) from baseline in PANSS total score with asenapine and olanzapine were similar at week 6 but showed a statistically significant difference in favor of olanzapine at endpoint. Whereas, in case of CGI-S score, between group differences were not significant but in case of CGI-I score there was small difference in favor of olanzapine [21].

In the present study, it was found that asenapine had significantly better outcome than olanzapine in case of BPRS, CGI-S, CGI-I scores. This may be due to the short duration of this study (12 weeks). Although our study reflected a better outcome in asenapine group of patients but the effectiveness of olanzapine could not be underestimated. Again the differences in the results may be due to some other related factors like differences in 
ethnicity, age and gender distribution, duration of study, sample size etc. Trial done by Schoemaker et al. [21] was done on 1,219 patients from 102 different sites from different continents all over the world and it was a flexible doses, 52 weeks phase III trial. There was no upper limit of age in their study and mean age was around 36 years. Where in present study age limit was $18-50$ years and mean age of recruited subjects were 31 years. Patients were also on concomitant medications like antidepressant, anxiolytics, hypnotics and others along with change of doses of study drugs were permissible in study done by Schoemaker et al. [21], which is not in concordance with present study.

Asenapine is a psychotropic agent with a unique receptor binding profile; it displays high affinity binding and antagonistic activity at a wide range of dopamine, serotonin, nor-adrenaline and histamine receptors. Asenapine has no appreciable affinity for muscarinic cholinergic receptors. It has been suggested that the efficacy of asenapine in schizophrenia is mediated through a combination of antagonistic activity at $\mathrm{D}_{2}$ and $5-\mathrm{HT}_{2 \mathrm{~A}}$ receptors [23-25]. Due to this unique action of asenapine, it elicited much more improvement in BPRS, CGI-S, CGI-I scores than olanzapine in the current study.

The safety parameters for the study were the changes in vital signs. The pulse rate, systolic and diastolic blood pressures were comparable at baseline and after treatment in both the medication groups. Considering lipid profile tests there were significant increase of post-treatment mean values of total cholesterol, triglyceride, LDL cholesterol, VLDL cholesterol and significant decrease of post treatment mean value of HDL cholesterol in both the treatment arms, although the mean values were well within normal limits. In case of between groups comparisons all the biochemical parameters were comparable in both the groups except serum total cholesterol, in which olanzapine increased post-treatment serum cholesterol more than asenapine. This reflects that patients having these medications should check their lipid profile once in 3 months to uncover any abnormality. The random blood glucose level at baseline and post-treatment was analogous both within and in-between the two treatment arms.

Schoemaker et al. [21] stated that there were no notable changes or between group differences in measures of total cholesterol, but triglyceride levels rose substantially with olanzapine and declined with asenapine. Whereas, the picture was quite different in the current study, which was may be due to biological variations and environmental factor $[26,27]$.

The incidences of adverse events were significantly more in olanzapine arm in which weight gain, Gl disturbances were more common. In the other hand, insomnia, somnolence, headache were frequently reported by asenapine treated patients. Incidences of anxiety were equal to both treatment arms. All the incidences were mild to moderate in nature.

In the study of Schoemaker et al. [21] using olanzapine as active comparator, it was observed that most reported adverse events were rated as mild or moderate. These adverse events included weight gain, insomnia, somnolence, gastrointestinal symptoms, akathisia. It was observed that change in weight gain was greater with olanzapine than asenapine [28]. Our present study corroborated with the above mentioned study. Although, there were some adverse events which were seen in only olanzapine group, like constipation, increased salivation and akathisia. None of the treatment emergent adverse events were severe enough to warrant withdrawal of the study medication. Nobody encountered any serious adverse events and there was no hospitalization.

Landbloom et al. [29] used asenapine in randomized, double-blind, fixed-dose, placebo controlled trial with olanzapine as an active control. This was the second head-to-head trial, where also asenapine proved its efficacy and safety. A case report published by Ochi et al. [30] in 2019, showed Efficacy of Asenapine in Schizophrenia patient resistant to Clozapine combined with Electroconvulsive Therapy. A severe treatment-refractory schizophrenic patient who did not respond to clozapine even with ECT, but recovered with asenapine monotherapy gradually.

The present study had certain limitations:

- Small sample size may have not shown a clear statistical difference between groups in other measures such as CGI-S and CGI-I rating.

- Study was single blind because we lacked the resources required for a truly double-blind study.

- Antipsychotic drugs are known to increase serum prolactin [24] but we could not measure prolactin levels because of logistic constraints.

- The treatment period in this study was relatively short (12 weeks), and hence provided no scope for 
assessing comparative effectiveness of long-term therapy or the detection of delayed adverse events.

- Cost effective analysis was also not done.

To conclude it can be said that newer atypical antipsychotic asenapine is more effective than the standard atypical antipsychotic olanzapine in reducing the symptoms of schizophrenia. Although asenapine is more efficacious but the safety profile would be kept in mind before prescribing it and lastly the cost of the treatment could be the major limitation of its use. Further studies on larger sample size and longer duration with cost analysis are required to establish the safety and efficacy of asenapine in our country.

\section{Acknowledgments}

We express our gratitude to all the faculty members, Post graduate trainees and staffs of Department of Pharmacology. We are also thankful to the all the Post graduate trainees, departmental staffs of Psychiatry Department and relatives of the recruited subjects.

\section{- Conflicts of Interest}

No potential conflict of interest relevant to this article was reported.

\section{Author Contributions}

Conceptualization: Arpita Maitra, Swati Bhattacharyya, and Sabyasachi Mukhopadhyay. Data acquisition: Arpita Maitra, Sabyasachi Mukhopadhyay, Asim Kumar Mallick, and Supreeti Biswas. Formal analysis: Arpita Maitra, Swati Bhattacharyya, Asim Kumar Mallick, and Om Prakash Singh. Funding: Arpita Maitra, Sabyasachi Mukhopadhyay, Supreeti Biswas. Supervision: Swati Bhattacharyya and Asim Kumar Mallick. Writing - original draft: Arpita Maitra, Swati Bhattacharyya, and Supreeti Biswas. Writing - editing and review: Arpita Maitra, Sabyasachi Mukhopadhyay, and Om Prakash Singh.

\section{ORCID}

Arpita Maitra https://orcid.org/0000-0001-9218-0843

Swati Bhattacharyya

https://orcid.org/0000-0002-1786-3772

Sabyasachi Mukhopadhyay https://orcid.org/0000-0001-5794-0480

Asim Kumar Mallick

https://orcid.org/0000-0001-6314-7470
Supreeti Biswas https://orcid.org/0000-0001-6560-0953

Om Prakash Singh https://orcid.org/0000-0001-7672-3676

\section{REFERENCES}

1. Reus VI. Mental disorders. In: Kasper DL, Fauci AS, Longo DL, Braunwald E, Hauser SL, Jameson JL, editors. Harrison's principles of internal medicine. 16th ed. New York:McGraw-Hill; 2005. p.2559-2560.

2. Lieberman JA, Stroup TS, McEvoy JP, Swartz MS, Rosenheck RA, Perkins DO, et al.; Clinical Antipsychotic Trials of Intervention Effectiveness (CATIE) Investigators. Effectiveness of antipsychotic drugs in patients with chronic schizophrenia. N Engl J Med 2005;353:1209-1223.

3. Harris EC, Barraclough B. Excess mortality of mental disorder. BrJ Psychiatry 1998;173:11-53.

4. Brown S. Excess mortality of schizophrenia. A meta-analysis. BrJ Psychiatry 1997; 171:502-508.

5. Thomas $\mathrm{S}$, editor. Drug reference for FDA approved psychiatric drugs [Internet]. Quincy (IL): Neurotransmitter.net; 2008 [cited at $2009 \mathrm{Feb}$ 1]. Available from: https://www. neurotransmitter.net/drug_reference.html.

6. Cerit C, Vural M, Bos Gelmez SÜ, Ozten E, Aker AT, Yıldız M. Metabolic syndrome with different antipsychotics: a multicentre cross-sectional study. Psychopharmacol Bull 2010;43: 22-36.

7. US Food and Drug Administration. 2004 safety alert on Zyprexa (olanzapine) [Internet]. Rockville (MD): US Food and Drug Administration MedWatch Programme; 2004 [cited at 2009 Feb 1]. Available from: https://www.fda.gov/files/drugs/published/Zyprexa-Medication-Guide.pdf.

8. Henry JM, Fuller MA. Asenapine: a new antipsychotic option. J Pharm Pract 2011;24:447-451.

9. Citrome L. Role of sublingual asenapine in treatment of schizophrenia. Neuropsychiatr Dis Treat 2011;7:325-339.

10. Kay SR, Fiszbein A, Opler LA. The positive and negative syndrome scale (PANSS) for schizophrenia. Schizophr Bull 1987; 13:261-276.

11. Overall JE, Gorham DR. The brief psychiatric rating scale. Psychol Rep 1962;10:799-812.

12. Leon AC, Shear MK, Klerman GL, Portera L, Rosenbaum JF, Goldenberg I. A comparison of symptom determinants of patient and clinician global ratings in patients with panic disorder and depression. J Clin Psychopharmacol 1993;13:327331.

13. Spearing MK, Post RM, Leverich GS, Brandt D, Nolen W. Modification of the Clinical Global Impressions (CGI) Scale for use in bipolar illness (BP): the CGI-BP. Psychiatry Res 1997;73:159-171.

14. Zaider TI, Heimberg RG, Fresco DM, Schneier FR, Liebowitz MR. Evaluation of the clinical global impression scale among individuals with social anxiety disorder. Psychol Med 2003; 33:611-622.

15. IBM Corp. The manual of IBM SPSS Statistics for Windows, 
Version 21.0. Armonk:IBM Corp;2012.

16. Abraha I, Montedori A. Modified intention to treat reporting in randomised controlled trials: systematic review. BMJ 2010; 340:c2697.

17. Lindenmayer JP, Khan A. Psychopathology. In: Lieberman JA, Stroup TS, Perkins DO, editors. The American Psychiatric Publishing textbook of schizophrenia. Washington, DC:American Psychiatric Publishing;2006. p.187-222.

18. Solmi M, Murru A, Pacchiarotti I, Undurraga J, Veronese N, Fornaro M, et al. Safety, tolerability, and risks associated with first- and second-generation antipsychotics: a state-of-the-art clinical review. Ther Clin Risk Manag 2017;13:757-777.

19. Conley RR, Kelly DL. Second-generation antipsychotics for schizophrenia: a review of clinical pharmacology and medication-associated side effects. Isr J Psychiatry Relat Sci 2005; 42:51-60.

20. Kane JM, Mackle M, Snow-Adami L, Zhao J, Szegedi A, Panagides J. A randomized placebo-controlled trial of asenapine for the prevention of relapse of schizophrenia after long-term treatment. J Clin Psychiatry 2011;72:349-355.

21. Schoemaker J, Naber D, Vrijland P, Panagides J, Emsley R. Long-term assessment of asenapine vs. olanzapine in patients with schizophrenia or schizoaffective disorder. Pharmacopsychiatry 2010;43:138-146.

22. Potkin SG, Cohen M, Panagides J. Efficacy and tolerability of asenapine in acute schizophrenia: a placebo- and risperidone-controlled trial. J Clin Psychiatry 2007;68:1492-1500.
23. Shahid M, Walker GB, Zorn SH, Wong EH. Asenapine: a novel psychopharmacologic agent with a unique human receptor signature. J Psychopharmacol 2009;23:65-73.

24. Weber J, McCormack PL. Asenapine. CNS Drugs 2009;23: 781-792.

25. Meyer JM. Pharmacotherapy of psychosis and mania. In: Brunton LL, Chabner BA, Knollmann BC, editors. Goodman \& Gilman's the pharmacological basis of therapeutics. 12th ed. New York:McGraw-Hill Medical; 2011. p.417-456.

26. Kalow W. Pharmacogenomics: historical perspective and current status. Methods Mol Biol 2005;311:3-15.

27. Kawanishi Y, Tachikawa H, Suzuki T. Pharmacogenomics and schizophrenia. Eur J Pharmacol 2000;410:227-241.

28. Simon V, van Winkel R, De Hert M. Are weight gain and metabolic side effects of atypical antipsychotics dose dependent? A literature review. J Clin Psychiatry 2009;70:1041-1050.

29. Landbloom R, Mackle M, Wu X, Kelly L, Snow-Adami L, McIntyre RS, et al. Asenapine for the treatment of adults with an acute exacerbation of schizophrenia: results from a randomized, double-blind, fixed-dose, placebo-controlled trial with olanzapine as an active control. CNS Spectr 2017; 22:333-341.

30. Ochi S, Inoue S, Yoshino Y, Shimizu H, Iga JI, Ueno SI. Efficacy of asenapine in schizophrenia resistant to clozapine combined with electroconvulsive therapy: a case report. Clin Psychopharmacol Neurosci 2019;17:559-563. 